\title{
Selection of Patients for Treatment of Brain Arteriovenous Malformations by the Transvenous Approach: Relationship with Venous Anatomy and Risk of Hemorrhagic Complications
}

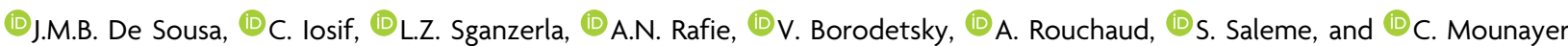

\begin{abstract}
BACKGROUND AND PURPOSE: Intracranial hemorrhage represents a severe complication of brain arteriovenous malformation treatment. The aim of this cohort was to report the rate of hemorrhagic complications after transvenous endovascular embolization and analyze the potential angioarchitectural risk factors as well as clinical outcomes.
\end{abstract}

MATERIALS AND METHODS: During an 11-year period, 57 patients underwent transvenous endovascular embolization. All cases of hemorrhagic complications were identified. We analyzed the following variables: sex, age, hemorrhagic presentation, SpetzlerMartin grade, size of the AVM before the transvenous treatment, number of venous collectors, pattern of drainage, presence of dilated veins, and technical aspects. Univariate and multivariate multiple regression analyses were performed to evaluate the potential risk factors for procedure-related hemorrhagic complications.

RESULTS: Hemorrhagic complications (either intraprocedural or periprocedural) unrelated to a perforation due to micronavigation occurred in $8(14.0 \%)$ procedures. Significant (mRS > 2) and persistent neurologic deficits were present in 2 (3.5\%) patients at 6month control. Larger nidi, especially $>3 \mathrm{~cm}(P=.03)$, and a larger number of venous collectors have shown a statistically significant correlation with hemorrhagic complications. Only the number of venous collectors was identified as an independent predictor of hemorrhagic complications in the multivariate analysis (OR, 8.7; 95\% confidence interval, 2.2-58.2) $(P=.006)$.

CONCLUSIONS: Larger nidus sizes and an increased number of venous collectors may increase the risk of hemorrhagic complications when implementing transvenous endovascular treatment of AVMs. The technique is effective and promising, especially with small nidi and single venous collectors.

ABBREVIATIONS: $\mathrm{bAVM}=$ brain arteriovenous malformation; $\mathrm{HC}=$ hemorrhagic complications; TVE $=$ transvenous endovascular embolization

E ndovascular treatment for brain arteriovenous malformations (bAVMs) was traditionally focused on targeting the nidus through an intra-arterial approach, whether as a means of curative treatment or targeted embolization as an adjunctive or emergency treatment. In an attempt to target parts of the nidus that lie in the deep brain areas or that are supplied by very thin or perforating arterial branches, transvenous endovascular embolization (TVE) has emerged and is becoming a new tool to target otherwise incurable brain AVMs. ${ }^{1-3}$

Even though TVE has several advantages, including a very high rate of angiographic cure up to $92.6 \%{ }^{3}$ and a means of

Received June 10, 2020; accepted after revision August 4.

From the Department of Interventional Neuroradiology, Hôpital Dupuytren, Centre Regional Hospitalier Universitaire de Limoges, Limoges, France.

Please address correspondence to Jorge Murilo Barbosa De Sousa, MD, MSc,

Department of Interventional Neuroradiology, Hôpital Dupuytren, Centre Regional Hospitalier Universitaire de Limoges, 2 Ave Martin Luther-King, 87042 Limoges

Cedex, France; e-mail: jorgemurilobs@gmail.com

http://dx.doi.org/10.3174/ajnr.A6810 curing otherwise incurable bAVMs, ${ }^{4,5}$ it still needs to be used very selectively, to reduce hemorrhagic complications (HC).

Nevertheless, the technique is not exempt from intraprocedural or periprocedural complications. Hemorrhagic complications represent the most important and challenging procedurerelated issues with this technique, which may lead to poor clinical outcomes. $^{6-9}$

A greater understanding of the frequency and risk factors for HC may optimize patient selection for TVE. The aim of this study was to estimate the rates of intraprocedural and periprocedural hemorrhage after TVE for bAVMs, to assess the morbidity and mortality associated with bleeding, and to analyze the angioarchitectural risk factors linked with such complications.

\section{MATERIALS AND METHODS Study Design and Participants}

The institutional review board and ethics committee of our institution had approved the current study protocol. All subjects (or 
legal guardians) signed an informed consent form. This cohort study was performed in accordance with the Code of Medical Ethics of the World Medical Association (Declaration of Helsinki, 2014).

We reviewed a prospectively collected data base of 192 patients with bAVMs who underwent endovascular treatment at our institution between January 2008 and July 2019. A total of 57 patients underwent TVE.

We assessed demographics, medical history, imaging results, postoperative examinations, and clinical follow-up among all patients. All $\mathrm{HC}$ were prospectively evaluated, and the location of the hemorrhage, its temporal relationship with TVE, clinical outcomes, mRS score, and imaging outcomes at discharge and after 6 months were recorded in a quantified fashion in a prospectively maintained data base.

The recorded anatomic data included the largest diameter of the nidus before any intervention, the diameter of the nidus immediately before TVE, the location of the nidus (cortical or deep), and the number of venous collectors. Three senior interventional neuroradiologists retrospectively evaluated whether draining veins were dilated or nondilated. The evaluator were also blinded to the outcome and a vein being dilated when it had at least twice the diameter of the corresponding contralateral vein. Additionally, we classified the nidi of bAVMs into 2 groups: those that were $0-3 \mathrm{~cm}$ in diameter and those of $>3 \mathrm{~cm}$ in diameter.

In addition to adult patients, children and adolescents with bAVMs treated by TVE were included.

Patients diagnosed with vein of Galen malformations and dural arteriovenous fistulas were excluded.

\section{Outcome Assessments}

In all cases, the goal of TVE was curative embolization of the bAVMs. Arterial embolization usually preceded the intervention, whenever possible, to reduce the size of nidus. However, for this series, we present only the clinical outcomes and complications of TVE.

Patients were examined by a senior neuroradiologist and a senior anesthesiologist. A CT scan was obtained immediately after each TVE session. As part of our protocol, cranial MR imaging was performed for all patients 24 hours before and after embolization.

The mRS score was used preoperatively and at follow-up assessment in a prospective fashion. The parameters assessed were the following: the overall frequency of $\mathrm{HC}$ (related and unrelated to endovascular access), the rate of obliteration at 6 months after treatment with TVE, and the existence of permanent neurologic deficits at 6 months. An adverse event was defined as a new neurologic deficit that occurred within 6 months of embolization. Angioarchitectural analyses considered only $\mathrm{HC}$ unrelated to endovascular access to avoid selection bias.

Follow-up angiograms were obtained at 6 months postembolization. Embolization was considered curative when obliteration of the nidus with no evidence of early venous drainage was demonstrated on an angiogram.

Follow-up data considered all patients, including those with incomplete endovascular therapy.

\section{Treatment}

Endovascular management was proposed for ruptured bAVMs as well as for unruptured bAVMs with high-risk angiographic findings, such as intranidal aneurysms and high-flow shunts and those presenting with symptoms suggestive of vascular flow steal phenomena.

TVE was performed as either a single TVE session or combined transarterial embolization and TVE. Ideally, the selection criteria for TVE included a nidus diameter of $<3 \mathrm{~cm}$ and a single draining vein. In cases with unfavorable anatomy for arterial embolization, including supply by tiny perforating arteries, "en passage" feeders, or no obvious arterial pedicle, a single TVE was performed. In cases in which partial arterial embolization was feasible, to reduce the nidus size, the embolization was initiated by the arterial side and was resumed by venous side embolization, to obtain complete occlusion of the nidus.

The complete technical description of TVE has been published elsewhere. ${ }^{3,5}$ All procedures were performed with the patient under heparinization. In TVE, a microcatheter was always placed into an arterial feeder, and a superselective angiogram was performed to obtain better visualization of the nidus, without overlapping vascular structures. This maneuver facilitated an optimal position of the venous microcatheter.

A microcatheter was placed as close as possible to the nidus (venous side), and ethylene-vinyl alcohol copolymer was slowly injected. On completion of the procedure, the microcatheter was cut at the level of the jugular sheath at all times. Arterial blood pressure was maintained at a maximum of $120 \times 80 \mathrm{~mm} \mathrm{Hg}$ after endovascular treatment or $20 \mathrm{~mm} \mathrm{Hg}$ lower than baseline tension in patients with previous hypertension. No further anticoagulant therapy was administered. After embolization, patients were kept in the intensive care unit for 48 hours.

To allow a continuous injection and a faster penetration of ethylene-vinyl alcohol copolymer in the nidus and minimize backflow inside the vein, we used coils on the venous side for veins of $\geq 5 \mathrm{~mm}$ in diameter, with low packing density and no glue. In these cases, a second microcatheter for coils was placed proximal to the tip of the first microcatheter.

\section{Statistical Analysis}

Statistical analysis was performed using R statistical and computing software, Version 3.6.1. (http://www.r-project.org/) and Excel for Mac, 2019 (Microsoft). Descriptive analysis was conducted to characterize the sample. Any missing data were described. Quantitative variables were expressed as the mean \pm $\mathrm{SD}$, and qualitative variables were expressed as frequency and percentage values. The normality of the data distribution was assessed using a Q-Q plot.

The Student $t$ test was used to compare continuous variables when normality was determined. In cases of rejection, the MannWhitney test was used. The Fisher exact test was used to compare qualitative data and frequency of occurrence. A statistical significance level of 5\% was used for all analyses.

Multiple linear regression analysis was conducted to identify the independent predictors among the clinical-anatomic-demographic data for the HC. For this analysis, all variables were initially considered. The selection process was performed using a stepwise algorithm. The strength of the relationship between HC and their correlates was interpreted using odds ratios with $95 \%$ confidence intervals. A $P$ value $<.05$ in the final model was 
considered statistically significant. In addition, calibration of the model was established using the Hosmer-Lemeshow goodness of fit test, as well as a calibration curve. The discriminative ability of the model was assessed by receiver operating characteristic curve analysis.

\section{RESULTS}

A total of 57 consecutive patients undergoing treatment for bAVMs by TVE were included. Clinical-demographic and anatomic variables are summarized in Table 1.

In $17(29.8 \%)$ patients, TVE was used exclusively, and in 40 patients $(70.2 \%)$, it was used in combination with the arterial approach (either during the same session or in previous sessions). The drainage vein was coiled in 17 (29.8\%) patients. An arterial balloon, aimed at obstructing and controlling flow at the nidus and facilitating the progression of embolic liquid through the vein, was used in $14(24.5 \%)$ patients.

Table 1: Clinical-demographic and anatomic variables of patients undergoing TVE for bAVMs

\begin{tabular}{lc}
\hline \multicolumn{1}{c}{ Variables } & Patients $(\boldsymbol{n}=\mathbf{5 7})$ \\
\hline Sex (No.) (\%) & \\
Male & $29(50.9 \%)$ \\
Female & $28(49.1 \%)$ \\
Age (mean) (range) (yr) & $18.1(9-78)$ \\
Previous rupture (No.) (\%) & $38(66.6 \%)$ \\
bAVM location (No.) (\%) & \\
Cortical (lobar or cerebellum) & $41(72 \%)$ \\
Deep (brain stem, thalamus, or basal ganglia) & $16(28.0 \%)$ \\
Nidus diameter (original size) (mean) (cm) & $2.91 \pm 1.26$ \\
Nidus diameter before treatment by TVE (mean) (cm) & $2.44 \pm 0.99$ \\
Dilated vein (No.) (\%) & \\
Evaluator 1 & $24(42.1 \%)$ \\
Evaluator 2 & $28(49.1 \%)$ \\
Evaluator 3 & $36(63.1 \%)$ \\
Pattern of venous drainage (No.) (\%) & \\
Superficial & $25(43.9 \%)$ \\
Deep & $28(49.1 \%)$ \\
Superficial and deep & $4(7.0 \%)$ \\
Venous collector (No.) (\%) & \\
I & $37(64.9 \%)$ \\
2 & $18(31.6 \%)$ \\
3 & $2(3.5 \%)$ \\
Spetzler-Martin grade & \\
I & $5(8.8 \%)$ \\
II & $18(31.6 \%)$ \\
III & $23(40.4 \%)$ \\
V & $10(17.5 \%)$ \\
\hline
\end{tabular}

Table 2: Functional outcome status of patients with hemorrhagic complications

\begin{tabular}{lccc}
\hline Patient & mRS before Treatment & mRS before Discharge & 6-Month Follow-Up mRS \\
\hline 1 & 0 & 0 & 0 \\
2 & 0 & 1 & NA \\
3 & 0 & 0 & 0 \\
4 & 1 & 4 & 3 \\
5 & 0 & 3 & 2 \\
6 & 1 & 2 & 1 \\
7 & 0 & 4 & 4 \\
8 & 0 & 2 & 1 \\
\hline
\end{tabular}

Note:- NA indicates not available.
A control angiogram was obtained in all patients. The anatomic cure rate was documented in 52 (91.2\%) bAVMs. We noticed total occlusion in $100 \%$ of the cases with Spetzler-Martin grades I, II, and III.

Only 1 patient missed follow-up (mRS score at 6 months) by moving to a different county. Specifically in this case, angiographic control was performed within 1 month.

\section{Outcomes and Hemorrhagic Complications}

We recorded 10 (17.5\%) overall hemorrhagic complications. One of these patients had subarachnoid hemorrhage due to intracranial dissection during arterial access in the combined approach. Another patient had perforation of the draining cortical vein during micronavigation. These 2 patients had no clinical consequences and were excluded from analysis of the $\mathrm{HC}$.

Thus, we analyzed a total of $8(14.0 \%) \mathrm{HC}$ with TVE, which was either intraprocedural or periprocedural. Three patients (5.2\%) had intraprocedural $\mathrm{HC} ; 3$ patients (5.2\%) had $\mathrm{HC}$ within 24 hours of the procedure; 1 patient $(1.7 \%)$, within 48 hours; and 1 patient (1.7\%), within 96 hours. No patient showed a hemorrhagic complication after this period.

Only 2 (3.5\%) patients had unfavorable outcomes (mRS score at 6 months of $>2$ ) at the 6-month follow-up (Table 2). Procedure-related mortality was $0 \%$.

Concerning the neuroimaging findings, of the 8 patients (14.0\%), 3 (5.2\%) had perinidal hematoma exclusively; 3 (5.2\%) had a hematoma with an intraventricular hemorrhage; 1 patient (1.7\%) had cortical subarachnoid hemorrhage; and 1 patient (1.7\%) had intraventricular hemorrhage. During the follow-up period, no recurrence was noted among the treated patients.

\section{Univariate and Multivariate Analyses}

Tables 3, 4, and 5 show the univariate and multivariate analyses performed to identify the independent predictors among the clinical, anatomic, demographic, and technical aspects of TVE data for the patients with HC. In univariate analysis, we found a statistically significant correlation between $\mathrm{HC}$ and the number of venous collectors, nidus diameter before any intervention, and nidus diameter immediately before treatment by TVE. No other significant associations were observed.

In the multiple linear regression analysis (Table 5), only the number of 
Table 3: Univariate analysis showing the association between findings on $\mathrm{HC}$ and the clinical-anatomic-demographic variables of patients treated by TVE

\begin{tabular}{|c|c|c|c|}
\hline \multirow[b]{2}{*}{ Variable } & \multicolumn{3}{|c|}{ Were There HC? } \\
\hline & No $(n=49)$ & Yes $(n=8)$ & $P$ \\
\hline Spetzler-Martin grading system & & & $1.0^{\mathrm{a}}$ \\
\hline I, II, and III & $39(68.5 \%)$ & 7 & \\
\hline IV and V & 10 (17.5\%) & 1 & \\
\hline Previous rupture & & & $1.0^{\mathrm{a}}$ \\
\hline No & 16 & 3 & \\
\hline Yes & 33 & 5 & \\
\hline Sex & & & $.70^{\mathrm{a}}$ \\
\hline Female & 25 & 3 & \\
\hline Male & 24 & 5 & \\
\hline Mean age on admission (SD) (yr) & $37.1(18.6)$ & $46.8(13.6)$ & $.16^{\mathrm{b}}$ \\
\hline No. of venous collectors (mean) (SD) & $1.2(0.45)$ & $2.0(0.75)$ & $.003^{\mathrm{C}}$ \\
\hline Presence of dilated draining vein (No.) (\%) & & & $.06^{\mathrm{a}}$ \\
\hline Evaluator $1=24 / 57$ & $18(31.5 \%)$ & $6(10.5 \%)$ & \\
\hline Presence of dilated draining vein (No.) (\%) & & & $.14^{\mathrm{a}}$ \\
\hline Evaluator $2=28 / 57$ & $22(38.5 \%)$ & $6(10.5 \%)$ & \\
\hline Presence of dilated draining vein (No.) (\%) & & & $.69^{\mathrm{a}}$ \\
\hline Evaluator $3=36 / 57$ & $30(52.6 \%)$ & $6(10.5 \%)$ & \\
\hline Pattern of venous drainage & & & $.48^{\mathrm{a}}$ \\
\hline Superficial & 21 & 4 & \\
\hline Deep & 25 & 3 & \\
\hline Superficial and deep & 3 & 1 & \\
\hline Nidus diameter before any intervention & & & $.01^{\mathrm{b}}$ \\
\hline (mean) (SD) (cm) & $2.74(1.19)$ & $3.88(1.40)$ & \\
\hline Nidus diameter before TVE (mean) (SD) (cm) & $2.28(0.92)$ & $3.37(1.00)$ & $.003^{b}$ \\
\hline Nidus diameter before TVE (2 groups) & & & $.03^{\mathrm{a}}$ \\
\hline $0-3 \mathrm{~cm}$ & 38 & 3 & \\
\hline$>3 \mathrm{~cm}$ & 11 & 5 & \\
\hline AVM location & & & $.09^{\mathrm{a}}$ \\
\hline Cortical (lobar or cerebellum) & 33 & 8 & \\
\hline Deep (brain stem, thalamus, or basal ganglia) & 16 & 0 & \\
\hline
\end{tabular}

Table 4: Univariate analysis showing the association between hemorrhagic complications and the technical variables of patients treated by TVE

\begin{tabular}{lccc}
\hline & \multicolumn{3}{c}{ Were There Hemorrhagic Complications? } \\
\cline { 2 - 4 } & No $(\boldsymbol{n}=49)$ & Yes $(\boldsymbol{n}=8)$ & $\boldsymbol{P}$ \\
\hline Arterial balloon & 11 & 3 & $.39^{\mathrm{a}}$ \\
Yes & 38 & 5 & \\
No & $1.8(1.2)$ & $1.8(0.99)$ & $.50^{\mathrm{b}}$ \\
No. of embolization sessions,(mean) (SD) & & & $1.0^{\mathrm{a}}$ \\
Coiled vein & 15 & 2 & \\
Yes & 34 & 6 & $1.0^{\mathrm{a}}$ \\
No & & & \\
Total occlusion (angiographic cure) & 44 & 8 & \\
Yes & 5 & 0 & \\
No & & & \\
\hline a Fisher exact test. & & & \\
${ }^{\mathrm{b}}$ Mann-Whitney test. &
\end{tabular}

Table 5: Multivariate logistic model with hemorrhagic complications as the binary end point $^{\mathrm{a}}$

\begin{tabular}{lccc}
\hline Variable & OR & $95 \%$ Cl & $P$ Value \\
\hline No. of venous collectors (unitary increase) & 8.72 & $2.2-58.2$ & $.006^{\mathrm{b}}$ \\
\hline${ }^{\mathrm{a}}$ Hosmer-Lemeshow goodness-of-fit test: $\chi^{2}=6.6, d f=8$, and $P$ value $=.57$. & & \\
b Significant at $P$-value $<.05$. & &
\end{tabular}

venous collectors still showed an association with $\mathrm{HC}(P=.006)$. The exponential coefficient of the number of venous collectors was 8.72 , which represented the odds ratio. This means that the existence of 1 more venous collector could increase the risk of $\mathrm{HC}$ by approximately 8.7 times ( $95 \%$ confidence interval, 2.2-58.2).

The Hosmer-Lemeshow goodness of fit test, which was not statistically significant $(P=.57)$, indicated that the predicted probability was in high concordance with the observed probability. The area under the receiver operating characteristic curve was 0.768 . The numeric value of the cutoff point for the predicted probability was 0.041 (cutoff for probability $=4.1 \%$ ). The sensitivity and specificity were $75.0 \%$ and $71.4 \%$, respectively. The negative and positive predictive values were $70.0 \%$ and $5.4 \%$, respectively.

\section{DISCUSSION}

To the best of our knowledge, this is the first publication with a specific focus on periprocedural HC in TVE of bAVMs. The angiographic cure rate using TVE in the present study has remained roughly the same since our recent publications. ${ }^{3,5}$ These studies reflected cure rates above $90 \%$, further reinforcing the effectiveness of this therapeutic technique for bAVMs.

In the study presented herein, hemorrhagic complications were observed in $14.0 \%$ of cases; nevertheless, unfavorable clinical outcomes ( $\mathrm{mRS}$ score at 6 months of $>2$ ) at 6 months were low (3.5\%). Fifty-nine percent of bAVMs (34 patients) in our series were SpetzlerMartin grade III or higher, thus yielding a higher risk of complications with all potential therapeutic alternatives. High Spetzler-Martin grades represent a challenge for all invasive therapeutic approaches. In neurosurgery, high Spetzler-Martin grades ( $\geq$ III) and deep brain localizations ${ }^{10}$ are related to high complication rates. ${ }^{11-14}$

Schaller et al ${ }^{15}$ reported a series of 150 patients undergoing microsurgery: the morbidity rate was $15.3 \%$, with permanent new deficits in $10.6 \%$. Another publication showed early neurologic deterioration after microsurgery in 
$39.2 \%$ of the 288 patients, among whom $12.2 \%$ had permanent deficits. $^{16}$

A literature review by Chen et $\mathrm{al}^{17}$ of 13 publications focused on TVE for bAVM (most of which were case reports) yielded an overall complication rate of $4.3 \%$. However, most of the 69 patients evaluated in that literature review were from our department. ${ }^{3}$

In another study of 408 patients treated by endovascular means with an arterial access, the rate of $\mathrm{HC}$ was 92 of 827 procedures (11.1\%); 48 complications (5.8\%) were unrelated to arterial perforation. ${ }^{18}$ In a meta-analysis that included a total of 98 publications and 8009 patients, periprocedural hemorrhage was observed after $2.6 \%$ of the transarterial embolization procedures for bAVMs, ${ }^{7}$ though cure rates with an arterial approach are lower than TVE. ${ }^{19}$

Given the high rate of angiographic cure in TVE embolization, a better understanding of HC may facilitate improved patient selection. Thus, we chose to perform univariate and multivariate analyses of only $\mathrm{HC}$ that were unrelated to access perforation.

In our univariate analysis, patients with larger nidi (either before any intervention or immediately before performance of TVE) had a significantly higher number of HC. In our view, larger bAVMs $(>3 \mathrm{~cm})$ are more likely to have $\mathrm{HC}$ for the following reasons: a larger nidus has a higher likelihood of a small hidden nidal remnant due to the superposition of the embolic agent and the subtraction process, which may fail to demonstrate a small remnant at the end of the procedure. Although all 8 patients had angiographic exclusion, ie, the absence of early venous drainage, in cases with a large nidus, the existence of a large cast of embolic liquid overlapping the nidal remnant is more probable.

Another potential factor is because there is greater difficulty in achieving complete distal occlusion of all arterial pedicles in cases with large nidi. Another possible reason, and not yet fully understood, is the phenomenon referred to as "delayed postoperative hemorrhage." 20 After embolization sessions, although the patients in the present study followed a strict blood pressure control protocol in an intensive care unit, we could not guarantee whether some of these patients experienced delayed postoperative hemorrhage. This is a condition of impaired cerebral vasoreactivity of the perinidal tissues, leading to edema and, in some cases, hemorrhage after exclusion of the artery-vein shunt. ${ }^{21,22}$

These results emphasize the necessity, whenever possible, of an initial arterial approach, to reduce the size of the nidus. This is common practice in our department, especially for bAVMs of $>3 \mathrm{~cm}$. Otherwise, TVE seems appropriate when bAVM remnants are not safely accessible by other therapeutic means.

Another variable that showed statistical significance with $\mathrm{HC}$ in the present study was the number of venous collectors in both univariate and multivariate analyses. The risk of $\mathrm{HC}$ increased by $8.7-$ fold for each additional venous collector unit $(P=.0061)$. Indeed, these results challenge the initial theory of the creators of the TVE technique, which was demonstrated by Massoud and Hademenos ${ }^{23}$ in animals. Those researchers stated that "these veins would act, in effect, as a safety valve to decompress the nidus in the event that the cumulative intranidal pressure becomes too high (eg, by an excessive retrograde injection pressure in the face of insufficient arterial hypotension)." 23
Our hypothesis is that in the presence of $>1$ venous collector, retrograde injection may induce inadvertent occlusion of the other drainage veins before complete occlusion of the nidus. This can then lead to increased intranidal pressure, rupture, and HC. This result has been well-demonstrated by Baharvahdat et $\mathrm{al}^{18}$ in a work on arterial embolization of $\mathrm{HC}$, in which early venous occlusion was significantly correlated with HC.

In addition to the reasons already mentioned, other hypotheses about non-access-related perforation $\mathrm{HC}$ include progressive venous congestion due to a slow flow and thrombosis and inflammatory reactions or mural necrosis induced by the embolic liquid. ${ }^{6,9,18,24}$

For TVE, our years of practice have shown that the best option for microcatheter removal is to cut it at the level of venous puncture, thereby avoiding unnecessary traction of fragile veins and an embolic material cast. ${ }^{3,5}$ Nevertheless, we cannot conclude that alternative techniques may lead to $\mathrm{HC}$ because in all such cases, cutting of the venous microcatheter was routinely performed.

When we compared our clinical outcomes with those of other bAVM embolization studies, only $3.5 \%$ of our patients had poor outcomes (mRS score at 6 months of $>2$ ) and no procedurerelated deaths were observed. In other studies of the arterial approach, we observed persistent symptoms after embolization, ranging from $3.8 \%$ to $14 \%{ }^{6,25,26}$ We believe that immediately after the procedure, neuroimaging is essential to evaluate all outcomes, in addition to intensive care and neurosurgical decompression when necessary. ${ }^{24,27}$

Nevertheless, our study provides adequate data to support the use of TVE in patients with bAVMs as an alternative in challenging cases or those with nidus remnants from previous therapeutic maneuvers. When deciding the appropriate treatment of bAVMs (ruptured or high-risk bAVMs), the ideal selection for TVE technique would be:

- Cases with single venous collector

- Nidi of $<3 \mathrm{~cm}$

- Deep-seated bAVMs.

In nidi of $>3 \mathrm{~cm}$ or bAVMs with $>1$ venous collector, TVE may be reasonable with prior arterial embolization. In addition, our study shows that dilated drainage veins should not deter the use of this treatment technique.

There are limitations to our study. First, the groups under evaluation were relatively small, precluding subset analysis, eg, of the technical aspects (Table 5), or showing no evidence of statistical significance. Second, although our data had been collected prospectively, data analysis was conducted retrospectively. The angiographic follow-up period was relatively short, and a longer duration would have promoted greater confidence in assessing anatomic obliteration. Furthermore, our study has other limitations that are common to cohort studies.

\section{CONCLUSIONS}

TVE seems to be a relatively safe and effective alternative for challenging bAVMs or bAVM remnants, especially when rapid cure is needed. In the presented study, a single venous collector, nidi $<3 \mathrm{~cm}$, and deeply seated bAVMs seemed to yield fewer hemorrhagic complications when TVE is used. Even though larger 
studies are needed, these factors should be considered in the patient selection for this technique and should be further investigated.

\section{ACKNOWLEDGMENTS}

We thank Angelo da Silva Cabral for assistance in statistical analysis of the study data.

\section{REFERENCES}

1. Choudhri O, Ivan ME, Lawton MT. Transvenous approach to intracranial arteriovenous malformations: challenging the axioms of arteriovenous malformation therapy? Neurosurgery 2015;77:644-51 CrossRef Medline

2. Mendes GA, Iosif C, Silveira EP, et al. Transvenous embolization in pediatric plexiform arteriovenous malformations. Neurosurgery 2016;78:45865 CrossRef Medline

3. Mendes GA, Kalani MY, Iosif C, et al. Transvenous curative embolization of cerebral arteriovenous malformations: a prospective cohort study. Neurosurgery 2018;83:957-64 CrossRef Medline

4. Consoli A, Renieri L, Nappini S, et al. Endovascular treatment of deep hemorrhagic brain arteriovenous malformations with transvenous Onyx embolization. AJNR Am J Neuroradiol 2013;34:180511 CrossRef Medline

5. Iosif C, Mendes GA, Saleme S, et al. Endovascular transvenous cure for ruptured brain arteriovenous malformations in complex cases with high Spetzler-Martin grades. J Neurosurg 2015;122:1229-38 CrossRef Medline

6. Haw CS, Terbrugge K, Willinsky R, et al. Complications of embolization of arteriovenous malformations of the brain. J Neurosurg 2006;104:226-32 CrossRef Medline

7. Subat YW, Dasenbrock HH, Gross BA, et al. Periprocedural intracranial hemorrhage after embolization of cerebral arteriovenous malformations: a meta-analysis. J Neurosurg 2019 Sep 13. [Epub ahead of print] CrossRef Medline

8. Ledezma CJ, Hoh BL, Carter BS, et al. Complications of cerebral arteriovenous malformation embolization: multivariate analysis of predictive factors. Neurosurgery 2006;58:602-11; discussion 602-11 CrossRef

9. Saatci I, Geyik S, Yavuz K, et al. Endovascular treatment of brain arteriovenous malformations with prolonged intranidal Onyx injection technique: long-term results in 350 consecutive patients with completed endovascular treatment course-clinical article. J Neurosurg 2011;115:78-88 CrossRef Medline

10. Potts MB, Jahangiri A, Jen M, et al; UCSF Brain AVM Study Project. Deep arteriovenous malformations in the basal ganglia, thalamus, and insula: multimodality management, patient selection, and results. World Neurosurg 2014;82:386-94 CrossRef Medline

11. Pan J, He H, Feng L, et al. Angioarchitectural characteristics associated with complications of embolization in supratentorial brain arteriovenous malformation. AJNR Am J Neuroradiol 2014;35:35459 CrossRef Medline
12. Heros RC. Brain resection for exposure of deep extracerebral and paraventricular lesions. Surg Neurol 1990;34:188-95 CrossRef Medline

13. Lawton MT, Hamilton MG, Spetzler RF. Multimodality treatment of deep arteriovenous malformations: thalamus, basal ganglia, and brain stem. Neurosurgery 1995;37:29-36 CrossRef Medline

14. Liu KD, Lee LS. Microsurgical treatment of deep arteriovenous malformations: basal ganglia and thalamus. Zhonghua Yi Xue Za Zhi (Taipei) 2001;64:23-30 Medline

15. Schaller C, Schramm J, Haun D. Significance of factors contributing to surgical complications and to late outcome after elective surgery of cerebral arteriovenous malformations. J Neurology Neurosurgery Psychiatry 1998;65:547-54 CrossRef Medline

16. Schramm J, Schaller K, Esche J, et al. Microsurgery for cerebral arteriovenous malformations: subgroup outcomes in a consecutive series of 288 cases. J Neurosurg 2017;126:1056-63 CrossRef Medline

17. Chen CJ, Norat P, Ding D, et al. Transvenous embolization of brain arteriovenous malformations: a review of techniques, indications, and outcomes. Neurosurg Focus 2018;45:E13-17 CrossRef

18. Baharvahdat $H$, Blanc $R$, Termechi R, et al. Hemorrhagic complications after endovascular treatment of cerebral arteriovenous malformations. AJNR Am J Neuroradiol 2014;35:978-83 CrossRef Medline

19. Elsenousi A, Aletich VA, Alaraj A. Neurological outcomes and cure rates of embolization of brain arteriovenous malformations with n-butyl cyanoacrylate or Onyx: a meta-analysis. J Neurointerv Surg 2016;8:265-72 CrossRef Medline

20. Morgan MK, Wiedmann MK, Assaad NN, et al. Deliberate employment of postoperative hypotension for brain arteriovenous malformation surgery and the incidence of delayed postoperative hemorrhage: a prospective cohort study. J Neurosurg 2017;127:1025-40 CrossRef Medline

21. Niini T, Laakso A, Tanskanen P, et al. Perioperative treatment of brain arteriovenous malformations between 2006 and 2014: the Helsinki Protocol. Neurocrit Care 2019;31:346-56 CrossRef Medline

22. Rangel-Castilla L, Spetzler RF, Nakaji P. Normal perfusion pressure breakthrough theory: a reappraisal after 35 years. Neurosurg Rev 2015;38:399-405 CrossRef Medline

23. Massoud TF, Hademenos GJ. Transvenous retrograde nidus sclerotherapy under controlled hypotension (TRENSH): a newly proposed treatment for brain arteriovenous malformations-Concepts and rationale. Neurosurgery 1999;45:351-65 CrossRef Medline

24. Cronqvist M, Wirestam R, Ramgren B, et al. Endovascular treatment of intracerebral arteriovenous malformations: procedural safety, complications, and results evaluated by MR imaging, including diffusion and perfusion imaging. AJNR Am J Neuroradiol 2006;27:16276 Medline

25. Hartmann A, Pile-Spellman J, Stapf C, et al. Risk of endovascular treatment of brain arteriovenous malformations. Stroke 2002;33:1816-20 CrossRef Medline

26. Meisel HJ, Mansmann U, Alvarez H, et al. Effect of partial targeted N-butyl-cyano-acrylate embolization in brain AVM. Acta Neurochir (Wien) 2002;144:879-88; discussion 888 CrossRef Medline

27. Cronqvist M, Ramgren B, Geijer B, et al. Diffusion- and perfusionweighted MRI in therapeutic neurointerventional procedures. Neuroradiology 2001;43:662-71 CrossRef Medline 